\title{
Partial pole assignment for the quadratic pencil by output feedback control with feedback designs
}

\author{
Wen-Wei Lin ${ }^{1, \ddagger}$ and Jenn-Nan Wang ${ }^{2, *, \dagger}$ \\ ${ }^{1}$ Department of Mathematics, National Tsing-Hua University, Hsinchu 300, Taiwan \\ ${ }^{2}$ Department of Mathematics, National Taiwan University, Taipei 106, Taiwan
}

\begin{abstract}
SUMMARY
In this paper we study the partial pole assignment problem for the quadratic pencil by output feedback control where the output matrix is also a designing parameter. In addition, the input matrix is set to be the transpose of the output matrix. Under certain assumption, we give a solution to this partial pole assignment problem in which the unwanted eigenvalues are moved to desired values and all other eigenpairs remain unchanged. Copyright $(c) 2005$ John Wiley \& Sons, Ltd.
\end{abstract}

KEY WORDS: partial pole assignment; quadratic pencils; output feedback control; feedback designs

\section{INTRODUCTION}

Vibrating structures with feedback controls such as bridges, buildings, airplanes and automobiles, generated by finite-element methods, are most often modelled in a system of secondorder differential equations

$$
M \ddot{q}(t)+D \dot{q}(t)+K q(t)=B u(t)
$$

where $M, D$ and $K$ are symmetric matrices with $M$ being non-singular, which represent mass, damping and stiffness matrices, respectively. The time-dependent variable $q(t) \in \mathbb{R}^{n \times 1}$ is the position vector, $B \in \mathbb{R}^{n \times m}$ is the full rank control feedback matrix and $u(t) \in \mathbb{R}^{m \times 1}$ is the control vector. In addition, the output or measurement vector $y(t) \in \mathbb{R}^{r \times 1}$ is given by

$$
y(t)=C q(t)
$$

\footnotetext{
*Correspondence to: Jenn-Nan Wang, Department of Mathematics, National Taiwan University, Taipei 106, Taiwan.

†E-mail: jnwang@math.ntu.edu.tw

‡E-mail: wwlin@am.nthu.edu.tw

Contract/grant sponsor: Taiwan National Science Council
}

Copyright (c) 2005 John Wiley \& Sons, Ltd.

Received 24 July 2003

Revised 16 November 2004 
where $C$ is a real $r \times n$ output matrix. In discussing the feedback control, we usually assume that the control vector $u(t)$ is defined by the control law

$$
u(t)=F y(t)+G \dot{y}(t)
$$

where $F, G \in \mathbb{R}^{m \times r}$ are output feedback gain matrices.

It is known that the open-loop system (1) gives rise to an open-loop quadratic pencil $P(\lambda)=\lambda^{2} M+\lambda D+K$ which has $2 n$ eigenvalues, say $\left\{\lambda_{j}\right\}_{j=1}^{2 n}$ (counted with multiplicity). It is clear that the set $\left\{\lambda_{j}\right\}_{j=1}^{2 n}$ is closed under complex conjugation, that is, if $\lambda$ is an eigenvalue of the pencil $P(\lambda)$, then so is $\bar{\lambda}$. Substituting (2) and (3) into (1) yields

$$
M \ddot{q}(t)+(D-B G C) \dot{q}(t)+(K-B F C) q(t)=0
$$

Likewise, the closed-loop system (4) is related to a closed-loop quadratic pencil $P_{\mathrm{c}}(\lambda)=\lambda^{2} M+$ $\lambda(D-B G C)+(K-B F C)$. Let $\left\{\lambda_{j}\right\}_{j=1}^{k} \subset \sigma(P)$ be closed under complex conjugation and $\left\{\mu_{j}\right\}_{j=1}^{k}$ be a new set of complex conjugate numbers. Hereafter, $\sigma(Q)$ denotes the spectrum of the pencil $Q$ or the matrix $Q$. In control theory, it is a natural question to find output feedback gain matrices $F$ and $G$ such that the spectrum $\sigma\left(P_{\mathrm{c}}\right)=\left\{\left\{\mu_{j}\right\}_{j=1}^{k},\left\{\lambda_{j}\right\}_{j=k+1}^{2 n}\right\}$. In other words, one would like to modify the original open-loop system (1) by output feedback control so that eigenvalues $\left\{\lambda_{j}\right\}_{j=1}^{k}$ of $P$ are replaced by $\left\{\mu_{j}\right\}_{j=1}^{k}$ and the remaining spectrum of $P$ are unchanged. This problem is called the partial pole assignment by output feedback control.

Before proceeding our discussion, we would like to make some remarks on this partial pole assignment problem. In the extreme case where the output matrix $C$ is non-singular, this problem can be solved by techniques developed in Reference [1] (single-input) or [2] (multiple-input) (see also References $[3,4]$ ). In fact, in this situation the problem is equivalent to the pole assignment problem by state feedback control. Practically, we often have $\operatorname{rank}(C)<n$. For this case, the techniques in References [1,2] are not applicable. It should be mentioned that orthogonality relations for the quadratic pencil $P(\lambda)$ play essential roles in the aforementioned papers. But these relations do not work well in the output feedback case. In order to attack this partial pole assignment problem by output feedback control, we therefore consider $B$ and $C$ as design variables as well. Moreover, to reduce the numbers of design parameters, it is desirable to set $C=B^{\mathrm{T}}$. Namely, the input and output devices are placed at the same location.

On the other hand, it is easily seen that the eigenvectors of the quadratic pencil determine the shape of the response for the associated second-order system. Therefore, in dealing with the partial pole assignment problem, we are interested in not only keeping the wanted eigenvalues but preserving the associated eigenvectors. This is achievable by means of orthogonality relations for $P(\lambda)$ when we consider the state feedback control (see References $[1,2])$. However, this is not easy to get in the case of output feedback control with the rank of the output matrix less than $n$.

Another issue being taken into account is that in real applications we may only need to change a small number of unwanted eigenvalues which is far less than the size of the matrix. Consequently, we hope that the rank of the input design or the output design is comparable to the number of unwanted eigenvalues. In the realization of the control system, this requirement corresponds to the fact that we only have to measure a handful of output data. This is indeed favourable in practice. 
In view of the previous remarks, we propose the following partial pole assignment problem using feedback designs (PPAPFD) in this paper.

Problem formulation (PPAPFD)

Assume that $P(\lambda)=\lambda^{2} M+\lambda D+K$ is a symmetric quadratic open-loop pencil with eigenpairs $\left\{\left(\lambda_{j}, y_{j}\right)\right\}_{j=1}^{2 n}$, where $M$ is non-singular. Let $\left\{\mu_{j}\right\}_{j=1}^{k}$ be a set of complex conjugate numbers, where $k \ll n$. Then one would like to design a control feedback matrix $B$ and find output gain matrices $F$ and $G$ such that the closed-loop pencil

$$
P_{\mathrm{c}}(\lambda)=\lambda^{2} M+\lambda\left(D-B G B^{\mathrm{T}}\right)+\left(K-B F B^{\mathrm{T}}\right)
$$

possesses eigenpairs $\left\{\left(\mu_{j}, x_{j}\right)\right\}_{j=1}^{k},\left\{\left(\lambda_{j}, y_{j}\right)\right\}_{j=k+1}^{2 n}$, where $x_{j}$ is a priori determined from $y_{j}$ without actually computing the kernel of $P_{\mathrm{c}}\left(\mu_{j}\right)$, for $j=1, \ldots, 2 n$.

Our work is based on a recent article by Carvalho et al. [5] where the authors investigate the model correction problem for the second-order system. We want to emphasize that the model correction focuses on tuning up the analytic model so that the experimental and theoretical results are as close as possible. Therefore, it is necessary to preserve the symmetric structure of the system in the model correction problem. That is, all corrected mass, damping and stiffness matrices are symmetric. The pole assignment problem has a different philosophy. Its main point is to modify the system by placing suitable controllers and designing appropriate control laws. Also, it can be seen as a way of changing the dynamical behaviour of the system after the model correction process. One of its well-known applications is to stabilize the system. Thus, the closed-loop pencil (5) is not required to be a symmetric one, i.e. $F$ and $G$ are not necessarily symmetric matrices. Having this concept in mind, in this paper we aim to develop a method to solve the PPAPFD when the unwanted and assigned modes satisfy certain condition (see the condition $(\mathscr{C})$ in Section 2).

Previously, a version of symmetric partial eigenstructure assignment problem with feedback design has been studied in the literature $[6,7]$ in which they use the eigenstructure assignment idea to perform the model correction. It is not difficult to see that eigenvectors for the closed-loop pencil can not be arbitrarily selected. Thus, in our proposed problem, we do not attempt to assign eigenvectors for $P_{\mathrm{c}}$. Instead, we would like to keep all wanted eigenvectors and give an explicit description of eigenvectors related to assigned eigenvalues. It should be remarked that the methods presented in References [6,7] neither preserve the wanted eigenpairs nor provide detailed information about eigenvectors associated to assigned eigenvalues. A closed related partial eigenstructure assignment problem for secondorder systems by state feedback control were considered in Reference [8] in which the input matrix $B$ is also a design parameter. For other eigenstructure assignment problems for second-order systems with fixed input matrix, we referred readers to Reference [9] and references therein.

This paper is organized as follows. In Section 2, we develop a method to solve the PPAPFD when the unwanted and assigned modes satisfy the condition $(\mathscr{C})$. In Section 3 , the condition $(\mathscr{C})$ is discussed in further detail and a necessary and sufficient condition to $(\mathscr{C})$ is given under some generic assumptions. In Section 4 , we provide some numerical testing results. 


\section{SOLUTION TO THE PPAPFD}

In this section we will develop a method to solve the PPAPFD under a suitable condition. Unlike the approach used in Reference [5] where the unwanted eigenvalue is replaced by a new value one by one, in this paper we assign all unwanted eigenvalues to new values in one step. To begin, we assume as before that $P(\lambda)=\lambda^{2} M+\lambda D+K$ is a symmetric quadratic pencil and $M$ is non-singular and let $\sigma(P)=\left\{\lambda_{j}\right\}_{j=1}^{2 n}$. Assume that $\left\{\lambda_{j}\right\}_{j=1}^{k}$ are the unwanted eigenvalues which are closed under complex conjugation and will be replaced by a set of complex conjugate numbers $\left\{\mu_{j}\right\}_{j=1}^{k}$. Therefore, there exist two matrices $\Lambda_{k}, Y_{k} \in \mathbb{R}^{k \times k}$ such that $\sigma\left(\Lambda_{k}\right)=\left\{\lambda_{j}\right\}_{j=1}^{k}$ and

$$
M Y_{k} \Lambda_{k}^{2}+D Y_{k} \Lambda_{k}+K Y_{k}=0
$$

From now on, a matrix pair $(J, V)$ is called an eigenmatrix pair of $P$ if it satisfies

$$
M V J^{2}+D V J+K V=0
$$

In this paper, in order to focus on the pole assignment problem, we will not discuss the theoretical or numerical issue of the quadratic eigenvalue problem in detail. The interested reader is referred to a nice survey article [10]. Nevertheless, we would like to provide a possible scenario to compute the eigenmatrix pair $\left(\Lambda_{k}, Y_{k}\right)$ based on a Schur basis. To this end, we linearize the quadratic pencil $P(\lambda)$. Let us introduce two $2 n \times 2 n$ matrices

$$
A=\left[\begin{array}{cc}
0 & I_{n} \\
K & D
\end{array}\right] \text { and } B=\left[\begin{array}{cc}
I_{n} & 0 \\
0 & -M
\end{array}\right]
$$

Then the quadratic eigenvalue problem $P(\lambda) u=0$ is equivalent to the generalized eigenvalue problem $A v=\lambda B v$, where

$$
v=\left[\begin{array}{l}
u \\
\lambda u
\end{array}\right]
$$

Using a $Q Z$-algorithm, we can compute two orthogonal matrices $Q, Z \in \mathbb{R}^{2 n \times 2 n}$ such that

$$
Q^{\mathrm{T}} A Z=\left[\begin{array}{cc}
R_{11} & R_{12} \\
0 & R_{22}
\end{array}\right] \text { and } Q^{\mathrm{T}} B Z=\left[\begin{array}{cc}
S_{11} & S_{12} \\
0 & S_{22}
\end{array}\right]
$$

where $R_{11} \in \mathbb{R}^{k \times k}, \quad R_{22} \in \mathbb{R}^{(2 n-k) \times(2 n-k)}$ are quasi-upper-triangular and $S_{11} \in \mathbb{R}^{k \times k}, \quad S_{22} \in$ $\mathbb{R}^{(2 n-k) \times(2 n-k)}$ are upper-triangular. We now write

$$
Z=\left[\begin{array}{ll}
Z_{11} & Z_{12} \\
Z_{21} & Z_{22}
\end{array}\right] \text { and } Q=\left[\begin{array}{ll}
Q_{11} & Q_{12} \\
Q_{21} & Q_{22}
\end{array}\right]
$$

where $Z_{11}, Z_{21}, Q_{11}, Q_{21} \in \mathbb{R}^{n \times k}$. In view of (7)-(9), we have that

$$
\left[\begin{array}{c}
Z_{21} \\
K Z_{11}+D Z_{21}
\end{array}\right]=\left[\begin{array}{l}
Q_{11} R_{11} \\
Q_{21} R_{11}
\end{array}\right] \text { and }\left[\begin{array}{c}
Z_{11} \\
-M Z_{21}
\end{array}\right]=\left[\begin{array}{l}
Q_{11} S_{11} \\
Q_{21} S_{11}
\end{array}\right]
$$


We note that $S_{11}$ is non-singular since $M>0$. Then it follows from (10) that

$$
K Z_{11}+D Z_{11}\left(S_{11}^{-1} R_{11}\right)+M Z_{11}\left(S_{11}^{-1} R_{11}\right)^{2}=0
$$

Now let $Z_{11}=Y_{k} T_{K}$ with $Y_{k}^{\mathrm{T}} Y_{k}=I_{k}$ (Schur basis), where $T_{k} \in \mathbb{R}^{k \times k}$ is non-singular uppertriangular. Then (11) becomes

$$
M Y_{k} \Lambda_{k}^{2}+D Y_{k} \Lambda_{k}+K Y_{k}=0
$$

where $\Lambda_{k}=T_{k}\left(S_{11}^{-1} R_{11}\right) T_{k}^{-1}$ is quasi-upper-triangular, i.e. a Schur form. Without loss of generality, we assume that $\Lambda_{k}$ is non-singular, equivalently, all eigenvalues $\left\{\lambda_{j}\right\}_{j=1}^{k}$ are non-zero. It is not difficult to see that this property can always be achieved by shifting the eigenvalues of $P(\lambda)$ (see Reference [1]).

The following condition is crucial in our approach to solve the PPAPFD. We assume that there exists $E_{k} \in \mathbb{R}^{k \times k}$ such that $I_{k}-E_{k} \Theta_{k}$ is non-singular and $\left\{\mu_{j}\right\}_{j=1}^{k}$ are eigenvalues of the matrix pair $\left(\Lambda_{k}-E_{k} \Lambda_{k}^{-\mathrm{T}} \Phi_{k}, I_{k}-E_{k} \Theta_{k}\right)$

where $\Phi_{k}=Y_{k}^{\mathrm{T}} K Y_{k}$ and $\Theta_{k}=Y_{k}^{\mathrm{T}} M Y_{k}$. It turns out that condition $(\mathscr{C})$ serves as a criterion of solving the PPAPFD when we intend to replace $\left\{\lambda_{j}\right\}_{j=1}^{k}$ by $\left\{\mu_{j}\right\}_{j=1}^{k}$. It should be noted that the invertibility of $I_{k}-E_{k} \Theta_{k}$ guarantees that the matrix pair $\left(\Lambda_{k}-E_{k} \Lambda_{k}^{-\mathrm{T}} \Phi_{k}, I_{k}-E_{k} \Theta_{k}\right)$ is not a singular pair. That is, the condition $(\mathscr{C})$ implies that

$$
\operatorname{det}\left(\lambda\left(I_{k}-E_{k} \Theta_{k}\right)-\left(\Lambda_{k}-E_{k} \Lambda_{k}^{-\mathrm{T}} \Phi_{k}\right)\right)=c \Pi_{j=1}^{k}\left(\lambda-\mu_{j}\right) \text { for some } c \neq 0
$$

At the first look, condition $(\mathscr{C})$ seems artificial and hard to verify. Fortunately, in some generic case, we can derive an easy sufficient condition on $\left(\Lambda_{k}, Y_{k}\right)$ such that $(\mathscr{C})$ is satisfied for almost all $\left\{\mu_{j}\right\}_{j=1}^{k}$ and $E_{k}$ can be easily constructed. We will discuss the condition ( $\left.\mathscr{C}\right)$ in further detail in the next section. With the condition $(\mathscr{C})$, we now can prove the following theorem.

\section{Theorem 2.1}

Assume that $\left\{\mu_{j}\right\}_{j=1}^{k}$ is a set of complex conjugate numbers such that condition $(\mathscr{C})$ holds with some real matrix $E_{k}$. Define

$$
\begin{aligned}
\tilde{M} & =M-M Y_{k} E_{k} Y_{k}^{\mathrm{T}} M \\
\tilde{D} & =D+M Y_{k} \Lambda_{k} H_{k} Y_{k}^{\mathrm{T}} K+K Y_{k} H_{k} \Lambda_{k}^{\mathrm{T}} Y_{k}^{\mathrm{T}} M \\
\tilde{K} & =K-K Y_{k} H_{k} Y_{k}^{\mathrm{T}} K
\end{aligned}
$$

where $H_{k}=\Lambda_{k}^{-1} E_{k} \Lambda_{k}^{-\mathrm{T}}$. Then the real quadratic pencil $\tilde{P}(\lambda)=\lambda^{2} \tilde{M}+\lambda \tilde{D}+\tilde{K}$ has the following properties:

(i) $\sigma(\tilde{P})=\left(\sigma(P) \backslash\left\{\lambda_{j}\right\}_{j=1}^{k}\right) \cup\left\{\mu_{j}\right\}_{j=1}^{k}$.

(ii) If $(\Omega, Z)$ is any eigenmatrix pair of $P(\lambda)$ satisfying $\sigma(\Omega) \cap \sigma\left(\Lambda_{k}\right)=\emptyset$, then $(\Omega, Z)$ is also an eigenmatrix pair of $\tilde{P}(\lambda)$, i.e.

$$
\tilde{M} Z \Omega^{2}+\tilde{D} Z \Omega+\tilde{K} Z=0
$$


Proof

(i) Since $\left(\Lambda_{k}, Y_{k}\right)$ satisfies $M Y_{k} \Lambda_{k}^{2}+D Y_{k} \Lambda_{k}+K Y_{k}=0$, we have that

$$
P(\lambda) Y_{k}=\left(\lambda^{2} M+\lambda D+K\right) Y_{k}=\left(M Y_{k}\left(\Lambda+\Lambda_{k}\right)+D Y_{k}\right)\left(\Lambda-\Lambda_{k}\right)
$$

where $\Lambda=\lambda I_{k}$. Now combining (12), the relation

$$
\operatorname{det}\left(I_{n}+R S\right)=\operatorname{det}\left(I_{m}+S R\right) \quad \text { for } \quad R \in \mathbb{C}^{n \times m} \text { and } S \in \mathbb{C}^{m \times n}
$$

and the condition $(\mathscr{C})$, we obtain that

$$
\begin{aligned}
\operatorname{det}(\tilde{P}(\lambda))= & \operatorname{det}\left(\lambda^{2} \tilde{M}+\lambda \tilde{D}+\tilde{K}\right) \\
= & \operatorname{det}\left(P(\lambda)-\lambda^{2} M Y_{k} E_{k} Y_{k}^{\mathrm{T}} M+\lambda K Y_{k} H_{k} \Lambda_{k}^{\mathrm{T}} Y_{k}^{\mathrm{T}} M+\lambda M Y_{k} \Lambda_{k} H_{k} Y_{k}^{\mathrm{T}} K\right. \\
& \left.-K Y_{k} H_{k} Y_{k}^{\mathrm{T}} K\right) \\
= & \operatorname{det}\left(P(\lambda)+\left(D Y_{k}+M Y_{k}\left(\Lambda+\Lambda_{k}\right)\right) \Lambda_{k} H_{k}\left(Y_{k}^{\mathrm{T}} K-\lambda \Lambda_{k}^{\mathrm{T}} Y_{k}^{\mathrm{T}} M\right)\right) \\
= & \operatorname{det}\left(P(\lambda)+P(\lambda) Y_{k}\left(\Lambda-\Lambda_{k}\right)^{-1} \Lambda_{k} H_{k}\left(Y_{k}^{\mathrm{T}} K-\lambda \Lambda_{k}^{\mathrm{T}} Y_{k}^{\mathrm{T}} M\right)\right) \\
= & \operatorname{det}(P(\lambda)) \operatorname{det}\left(I_{k}+\left(\Lambda-\Lambda_{k}\right)^{-1} \Lambda_{k} H_{k}\left(\Phi_{k}-\lambda \Lambda_{k}^{\mathrm{T}} \Theta_{k}\right)\right) \\
= & \operatorname{det}(P(\lambda))\left\{\Pi_{j=1}^{k}\left(\lambda-\lambda_{j}\right)\right\}^{-1} \operatorname{det}\left(\lambda\left(I_{k}-\Lambda_{k} H_{k} \Lambda_{k}^{\mathrm{T}} \Theta_{k}\right)-\left(\Lambda_{k}-\Lambda_{k} H_{k} \Phi_{k}\right)\right) \\
= & \operatorname{det}(P(\lambda))\left\{\Pi_{j=1}^{k}\left(\lambda-\lambda_{j}\right)\right\}^{-1} \operatorname{det}\left(\lambda\left(I_{k}-E_{k} \Theta_{k}\right)-\left(\Lambda_{k}-E_{k} \Lambda_{k}^{-\mathrm{T}} \Phi_{k}\right)\right) \\
= & \operatorname{det}(P(\lambda))\left\{\Pi_{j=1}^{k}\left(\lambda-\lambda_{j}\right)\right\}^{-1}\left\{c \Pi_{j=1}^{k}\left(\lambda-\mu_{j}\right)\right\}
\end{aligned}
$$

for some constant $c \neq 0$. In the above computations, we remind the reader that $E_{k}=\Lambda_{k} H_{k} \Lambda_{k}^{\mathrm{T}}$. Therefore, $\tilde{P}(\lambda)$ has the same eigenvalues as $P(\lambda)$, except that $\left\{\lambda_{j}\right\}_{j=1}^{k}$ are substituted by $\left\{\mu_{j}\right\}_{j=1}^{k}$.

(ii) Since $\left(\Lambda_{k}, Y_{k}\right)$ and $(\Omega, Z)$ are eigenmatrix pairs of $P$, we get that

$$
\begin{aligned}
\Lambda_{k}^{\mathrm{T}} Y_{k}^{\mathrm{T}} M Z \Omega^{2}+\Lambda_{k}^{\mathrm{T}} Y_{k}^{\mathrm{T}} D Z \Omega+\Lambda_{k}^{\mathrm{T}} Y_{k}^{\mathrm{T}} K Z & =0 \\
\left(\Lambda_{k}^{\mathrm{T}}\right)^{2} Y_{k}^{\mathrm{T}} M Z \Omega+\Lambda_{k}^{\mathrm{T}} Y_{k}^{\mathrm{T}} D Z \Omega+Y_{k}^{\mathrm{T}} K Z \Omega & =0
\end{aligned}
$$

Eliminating the common term in these two equalities gives

$$
\left(\Lambda_{k}^{\mathrm{T}} Y_{k}^{\mathrm{T}} M Z \Omega-Y_{k}^{\mathrm{T}} K Z\right) \Omega-\Lambda_{k}^{\mathrm{T}}\left(\Lambda_{k}^{\mathrm{T}} Y_{k}^{\mathrm{T}} M Z \Omega-Y_{k}^{\mathrm{T}} K Z\right)=0
$$

which immediately leads to

$$
\Lambda_{k}^{\mathrm{T}} Y_{k}^{\mathrm{T}} M Z \Omega=Y_{k}^{\mathrm{T}} K Z
$$


whenever $\sigma(\Omega) \cap \sigma\left(\Lambda_{k}\right)=\emptyset$. By virtue of (14), we have that

$$
\begin{aligned}
& \tilde{M} Z \Omega^{2}+\tilde{D} Z \Omega+\tilde{K} Z \\
&=M Z \Omega^{2}+D Z \Omega+K Z-M Y_{k} E_{k} Y_{k}^{\mathrm{T}} M Z \Omega^{2}+M Y_{k} \Lambda_{k} H_{k} Y_{k}^{\mathrm{T}} K Z \Omega+K Y_{k} H_{k} \Lambda_{k}^{\mathrm{T}} Y_{k}^{\mathrm{T}} M Z \Omega \\
&-K Y_{k} H_{k} Y_{k}^{\mathrm{T}} K Z \\
&=-M Y_{k} \Lambda_{k} H_{k} \Lambda_{k}^{\mathrm{T}} Y_{k}^{\mathrm{T}} M Z \Omega^{2}+M Y_{k} \Lambda_{k} H_{k} Y_{k}^{\mathrm{T}} K Z \Omega+K Y_{k} H_{k} \Lambda_{k}^{\mathrm{T}} Y_{k}^{\mathrm{T}} M Z \Omega-K Y_{k} H_{k} Y_{k}^{\mathrm{T}} K Z \\
&=-M Y_{k} \Lambda_{k} H_{k}\left(\Lambda_{k}^{\mathrm{T}} Y_{k}^{\mathrm{T}} M Z \Omega-Y_{k}^{\mathrm{T}} K Z\right) \Omega+K Y_{k} H_{k}\left(\Lambda_{k}^{\mathrm{T}} Y_{k}^{\mathrm{T}} M Z \Omega-Y_{k}^{\mathrm{T}} K Z\right) \\
&= 0
\end{aligned}
$$

Theorem 2.1 implies that the unwanted eigenvalues $\left\{\lambda_{j}\right\}_{j=1}^{k}$ of $P(\lambda)$ are replaced by $\left\{\mu_{j}\right\}_{j=1}^{k}$ in $\tilde{P}(\lambda)$. Moreover, all wanted eigenpairs $\left\{\left(\lambda_{j}, y_{j}\right)\right\}_{j=k+1}^{2 n}$ of $P(\lambda)$ remain as eigenpairs of $\tilde{P}(\lambda)$. Next we want to investigate the relationship between the eigenvectors of $\tilde{P}(\lambda)$ associated with $\left\{\mu_{j}\right\}_{j=1}^{k}$ and the unwanted eigenvectors $\left\{y_{j}\right\}_{j=1}^{k}$ of $P(\lambda)$.

Theorem 2.2

Assume that $(\mathscr{C})$ is satisfied with a suitable $E_{k}$. Let

$$
\Gamma_{k}:=\left(I_{k}-E_{k} \Theta_{k}\right)^{-1}\left(\Lambda_{k}-E_{k} \Lambda_{k}^{-\mathrm{T}} \Phi_{k}\right)
$$

then $\left(\Gamma_{k}, Y_{k}\right)$ is an eigenmatrix pair of $\tilde{P}(\lambda)$, i.e.

$$
\tilde{M} Y_{k} \Gamma_{k}^{2}+\tilde{D} Y_{k} \Gamma_{k}+\tilde{K} Y_{k}=0
$$

Proof

By virtue of $M Y_{k} \Lambda_{k}^{2}+D Y_{k} \Lambda_{k}+K Y_{k}=0$, we can derive that

$$
\begin{aligned}
D & Y_{k} \Gamma_{k}+K Y_{k}\left(H_{k} \Lambda_{k}^{\mathrm{T}} \Theta_{k} \Gamma_{k}+I_{k}-H_{k} \Phi_{k}\right) \\
& =D Y_{k} \Gamma_{k}+\left(-M Y_{k} \Lambda_{k}^{2}-D Y_{k} \Lambda_{k}\right)\left(H_{k} \Lambda_{k}^{\mathrm{T}} \Theta_{k} \Gamma_{k}+I_{k}-H_{k} \Phi_{k}\right) \\
& =-M Y_{k} \Lambda_{k}^{2}\left(H_{k} \Lambda_{k}^{\mathrm{T}} \Theta_{k} \Gamma_{k}+I_{k}-H_{k} \Phi_{k}\right)+D Y_{k}\left(\Gamma_{k}-\Lambda_{k} H_{k} \Lambda_{k}^{\mathrm{T}} \Theta_{k} \Gamma_{k}-\Lambda_{k}+\Lambda_{k} H_{k} \Phi_{k}\right) \\
& =-M Y_{k} \Lambda_{k}^{2}\left(H_{k} \Lambda_{k}^{\mathrm{T}} \Theta_{k} \Gamma_{k}+I_{k}-H_{k} \Phi_{k}\right)+D Y_{k}\left(\left(I_{k}-E_{k} \Theta_{k}\right) \Gamma_{k}-\left(\Lambda_{k}-E_{k} \Lambda_{k}^{-\mathrm{T}} \Phi_{k}\right)\right) \\
& =-M Y_{k} \Lambda_{k}^{2}\left(H_{k} \Lambda_{k}^{\mathrm{T}} \Theta_{k} \Gamma_{k}+I_{k}-H_{k} \Phi_{k}\right)
\end{aligned}
$$


It follows from (15) that

$$
\begin{aligned}
\tilde{M} Y_{k} & \Gamma_{k}^{2}+\tilde{D} Y_{k} \Gamma_{k}+\tilde{K} Y_{k} \\
= & \left(M-M Y_{k} E_{k} Y_{k}^{\mathrm{T}} M\right) Y_{k} \Gamma_{k}^{2}+\left(D+M Y_{k} \Lambda_{k} H_{k} Y_{k}^{\mathrm{T}} K+K Y_{k} H_{k} \Lambda_{k}^{\mathrm{T}} Y_{k}^{\mathrm{T}} M\right) Y_{k} \Gamma_{k} \\
& +\left(K-K Y_{k} H_{k} Y_{k}^{\mathrm{T}} K\right) Y_{k} \\
= & M Y_{k}\left(\Gamma_{k}^{2}-E_{k} \Theta_{k} \Gamma_{k}^{2}+\Lambda_{k} H_{k} \Phi_{k} \Gamma_{k}\right)+D Y_{k} \Gamma_{k}+K Y_{k}\left(H_{k} \Lambda_{k}^{\mathrm{T}} \Theta_{k} \Gamma_{k}+I_{k}-H_{k} \Phi_{k}\right) \\
= & M Y_{k}\left(\Gamma_{k}^{2}-E_{k} \Theta_{k} \Gamma_{k}^{2}+\Lambda_{k} H_{k} \Phi_{k} \Gamma_{k}-\Lambda_{k}^{2} H_{k} \Lambda_{k}^{\mathrm{T}} \Theta_{k} \Gamma_{k}-\Lambda_{k}^{2}+\Lambda_{k}^{2} H_{k} \Phi_{k}\right) \\
= & M Y_{k}\left(\left(\left(I_{k}-E_{k} \Theta_{k}\right) \Gamma_{k}-\left(\Lambda_{k}-E_{k} \Lambda_{k}^{-\mathrm{T}} \Phi_{k}\right)\right) \Gamma_{k}+\Lambda_{k}\left(\left(I_{k}-E_{k} \Theta_{k}\right) \Gamma_{k}-\left(\Lambda_{k}-E_{k} \Lambda_{k}^{-\mathrm{T}} \Phi_{k}\right)\right)\right. \\
= & 0
\end{aligned}
$$

Therefore, if $\Gamma_{k}$ is diagonalizable, i.e.

$$
\operatorname{diag}\left(\mu_{1}, \ldots, \mu_{k}\right)=Q_{k}^{-1} \Gamma_{k} Q_{k} \quad \text { for some non-singular matrix } Q_{k} \in \mathbb{C}^{k \times k}
$$

then $x_{j}=Y_{k} Q_{k} e_{j}$ is an eigenvector of $\tilde{P}\left(\mu_{j}\right)$ for $1 \leqslant j \leqslant k$, where $e_{j}$ is the $j$ th column of the identity matrix $I_{k}$.

Now, to solve the PPAPFD, we need to convert the quadratic pencil $\tilde{P}(\lambda)$ into the closedloop pencil $P_{\mathrm{c}}(\lambda)$ as in (5) with appropriate $B, F$ and $G$. In view of (13) and the invertibility of $\left(I_{k}-E_{k} \Theta_{k}\right)$, we can see that

$$
\operatorname{det}\left(I_{n}-M Y_{k} E_{k} Y_{k}^{\mathrm{T}}\right)=\operatorname{det}\left(I_{k}-E_{k} \Theta_{k}\right) \neq 0
$$

i.e. $I_{n}-M Y_{k} E_{k} Y_{k}^{\mathrm{T}}$ is invertible. Now the inverse of $I_{n}-M Y_{k} E_{k} Y_{k}^{\mathrm{T}}$ is given by the ShermanMorrison-Woodbury formula

$$
\left(I_{n}-M Y_{k} E_{k} Y_{k}^{\mathrm{T}}\right)^{-1}=I_{n}+M Y_{k} E_{k}\left(I_{k}-\Theta_{k} E_{k}\right)^{-1} Y_{k}^{\mathrm{T}}
$$

Notice that $I_{k}-\Theta_{k} E_{k}$ is invertible. To get $P_{\mathrm{c}}(\lambda)$, we set $P_{\mathrm{c}}(\lambda)=\left(I_{n}-M Y_{k} E_{k} Y_{k}^{\mathrm{T}}\right)^{-1} \tilde{P}(\lambda)$. Hence, using $D Y_{k}=-M Y_{k} \Lambda_{k}-K Y_{k} \Lambda_{k}^{-1}$, we have that

$$
\begin{aligned}
P_{\mathrm{c}}(\lambda)= & \left(I_{n}-M Y_{k} E_{k} Y_{k}^{\mathrm{T}}\right)^{-1}\left(\lambda^{2} \tilde{M}+\lambda \tilde{D}+\tilde{K}\right) \\
= & \lambda^{2} M+\lambda\left(I_{n}+M Y_{k} E_{k}\left(I_{k}-\Theta_{k} E_{k}\right)^{-1} Y_{k}^{\mathrm{T}}\right)\left(D+M Y_{k} \Lambda_{k} H_{k} Y_{k}^{\mathrm{T}} K+K Y_{k} H_{k} \Lambda_{k}^{\mathrm{T}} Y_{k}^{\mathrm{T}} M\right) \\
& +\left(I_{n}+M Y_{k} E_{k}\left(I_{k}-\Theta_{k} E_{k}\right)^{-1} Y_{k}^{\mathrm{T}}\right)\left(K-K Y_{k} H_{k} Y_{k}^{\mathrm{T}} K\right) \\
= & \lambda^{2} M+\lambda\left[D+M Y_{k} E_{k}\left(I_{k}-\Theta_{k} E_{k}\right)^{-1}\left(-\Lambda_{k}^{\mathrm{T}} Y_{k}^{\mathrm{T}} M-\Lambda_{k}^{-\mathrm{T}} Y_{k}^{\mathrm{T}} K\right)+M Y_{k} \Lambda_{k} H_{k} Y_{k}^{\mathrm{T}} K\right. \\
& +M Y_{k} E_{k}\left(I_{k}-\Theta_{k} E_{k}\right)^{-1} \Theta_{k} \Lambda_{k} H_{k} Y_{k}^{\mathrm{T}} K+K Y_{k} H_{k} \Lambda_{k}^{\mathrm{T}} Y_{k}^{\mathrm{T}} M \\
& \left.+M Y_{k} E_{k}\left(I_{k}-\Theta_{k} E_{k}\right)^{-1} \Phi_{k} H_{k} \Lambda_{k}^{\mathrm{T}} Y_{k}^{\mathrm{T}} M\right]+\left[K+M Y_{k} E_{k}\left(I_{k}-\Theta_{k} E_{k}\right)^{-1} Y_{k}^{\mathrm{T}} K\right.
\end{aligned}
$$




$$
\begin{aligned}
& \left.-K Y_{k} H_{k} Y_{k}^{\mathrm{T}} K-M Y_{k} E_{k}\left(I_{k}-\Theta_{k} E_{k}\right)^{-1} \Phi_{k} H_{k} Y_{k}^{\mathrm{T}} K\right] \\
= & \lambda^{2} M+\lambda\left(D-B G B^{\mathrm{T}}\right)+\left(K-B F B^{\mathrm{T}}\right)
\end{aligned}
$$

where

$$
G=\left[\begin{array}{cc}
E_{k}\left(I_{k}-\Theta_{k} E_{k}\right)^{-1}\left(I_{k}-\Phi_{k} H_{k}\right) \Lambda_{k}^{\mathrm{T}} & E_{k}\left(I_{k}-\Theta_{k}, K Y_{k}\right)^{-1}\left(\Lambda_{k}^{-\mathrm{T}}-\Theta_{k} \Lambda_{k} H_{k}\right)-\Lambda_{k} H_{k} \\
-H_{k} \Lambda_{k}^{\mathrm{T}} & 0
\end{array}\right]
$$

and

$$
F=\left[\begin{array}{cc}
0 & E_{k}\left(I_{k}-\Theta_{k} E_{k}\right)^{-1}\left(\Phi_{k} H_{k}-I_{k}\right) \\
0 & H_{k}
\end{array}\right]
$$

In summary, we have proved the following theorem which provides a solution to the PPAPFD.

Theorem 2.3

Let $\left\{\left(\lambda_{j}, y_{j}\right)\right\}_{j=1}^{k}$ eigenpairs of the open-loop pencil $P(\lambda)$ with $\Lambda_{k}$ and $Y_{k}$ defined in (6) and $\Lambda_{k}$ being non-singular, where $k<n / 2$. Assume that $\left\{\mu_{j}\right\}_{j=1}^{k}$ is a set of complex conjugate numbers satisfying condition $(\mathscr{C})$ with an appropriate matrix $E_{k}$. Then, by choosing the control matrix $B=\left[M Y_{k}, K Y_{k}\right]$ and gain matrices $G, F$ as in (17), (18), the closed-loop pencil $P_{\mathrm{c}}(\lambda)=\lambda^{2} M+$ $\lambda\left(D-B G B^{\mathrm{T}}\right)+\left(K-B F B^{\mathrm{T}}\right)$ has eigenpairs $\left\{\left(\mu_{j}, x_{j}\right)\right\}_{j=1}^{k} \cup\left\{\left(\lambda_{j}, y_{j}\right)\right\}_{j=k+1}^{2 n}$, where $x_{j}=Y_{k} Q_{k} e_{j}$ provided that $Q_{k}^{-1} \Gamma_{k} Q_{k}=\operatorname{diag}\left(\mu_{1}, \ldots, \mu_{k}\right)$.

\section{Remark 2.4}

The assumption of $k<n / 2$ ensures that $\operatorname{rank}(B)<n$. In the next section we will see that under some generic condition $\Gamma_{k}$ is always diagonalizable by choosing suitable $E_{k}$.

\section{DISCUSSION OF THE CONDITION $(\mathscr{C})$}

In this section we would like to discuss the condition $(\mathscr{C})$ in further detail. Our goal is to establish an equivalent condition of $(\mathscr{C})$ under some assumptions on the unwanted eigenmatrix pair $\left(\Lambda_{k}, Y_{k}\right)$, where $\Lambda_{k}$ is assumed to be non-singular.

\section{Theorem 3.1}

Let $\Theta_{k}$ and $\Lambda_{k}-\Theta_{k}^{-1} \Lambda_{k}^{-\mathrm{T}} \Phi_{k}$ be non-singular matrices. Then the set of complex conjugate numbers $\left\{\mu_{j}\right\}_{j=1}^{k}$ satisfies condition ( $\left.\mathscr{C}\right)$ if and only if $\Sigma_{k}-\Theta_{k}^{-1} \Lambda_{k}^{-\mathrm{T}} \Phi_{k}$ is non-singular for some $\Sigma_{k} \in \mathbb{R}^{k \times k}$ with $\sigma\left(\Sigma_{k}\right)=\left\{\mu_{j}\right\}_{j=1}^{k}$.

Proof

We first prove the necessity. Let $\left\{\mu_{j}\right\}_{j=1}^{k}$ satisfy $(\mathscr{C})$ with a matrix $E_{k}$. Since $\Theta_{k}$ is nonsingular, we set $E_{k}=\mathscr{E}_{k} \Theta_{k}^{-1}$ for some $\mathscr{E}_{k} \in \mathbb{R}^{k \times k}$, i.e. $E_{k} \Theta_{k}=\mathscr{E}_{k}$. Let

$$
\Sigma_{k}:=\left(I_{k}-\mathscr{E}_{k}\right)^{-1}\left(\Lambda_{k}-\mathscr{E}_{k} \Theta_{k}^{-1} \Lambda_{k}^{-\mathrm{T}} \Phi_{k}\right)
$$


then we have that $\sigma\left(\Sigma_{k}\right)=\left\{\mu_{j}\right\}_{j=1}^{k}$ and

$$
\mathscr{E}_{k}\left(\Sigma_{k}-\Theta_{k}^{-1} \Lambda_{k}^{-\mathrm{T}} \Phi_{k}\right)=\Sigma_{k}-\Lambda_{k}
$$

which leads to

$$
\Sigma_{k}-\Theta_{k}^{-1} \Lambda_{k}^{-\mathrm{T}} \Phi_{k}=\left(I-\mathscr{E}_{k}\right)^{-1}\left(\Lambda_{k}-\Theta_{k}^{-1} \Lambda_{k}^{-\mathrm{T}} \Phi_{k}\right)
$$

Therefore, $\Sigma_{k}-\Theta_{k}^{-1} \Lambda_{k}^{-\mathrm{T}} \Phi_{k}$ is non-singular.

Next we prove the sufficiency. Assume that there exists $\Sigma_{k} \in \mathbb{R}^{k \times k}$ with $\sigma\left(\Sigma_{k}\right)=\left\{\mu_{j}\right\}_{j=1}^{k}$ such that $\Sigma_{k}-\Theta_{k}^{-1} \Lambda_{k}^{-\mathrm{T}} \Phi_{k}$ is non-singular. Define

$$
\mathscr{E}_{k}=\left(\Sigma_{k}-\Lambda_{k}\right)\left(\Sigma_{k}-\Theta_{k}^{-1} \Lambda_{k}^{-\mathrm{T}} \Phi_{k}\right)^{-1}
$$

Then it is easily seen that

$$
I_{k}-\mathscr{E}_{k}=\left(\Lambda_{k}-\Theta_{k}^{-1} \Lambda_{k}^{-\mathrm{T}} \Phi_{k}\right)\left(\Sigma_{k}-\Theta_{k}^{-1} \Lambda_{k}^{-\mathrm{T}} \Phi_{k}\right)^{-1}
$$

and thus $I_{k}-\mathscr{E}_{k}$ is non-singular. Now it follows from (19) that $\left(I_{k}-\mathscr{E}_{k}\right)^{-1}\left(\Lambda_{k}-\mathscr{E}_{k} \Theta_{k}^{-1} \Lambda_{k}^{-\mathrm{T}} \Phi_{k}\right)$ $=\Sigma_{k}$ which immediately implies that $\left\{\mu_{j}\right\}_{j=1}^{k}$ are eigenvalues of the matrix pair $\left(\Lambda_{k}-\mathscr{E}_{k} \Theta_{k}^{-1} \Lambda_{k}^{-\mathrm{T}} \Phi_{k}, I_{k}-\mathscr{E}_{k}\right)$. Finally, we set $E_{k}=\mathscr{E}_{k} \Theta_{k}^{-1}$ and condition ( $\left.\mathscr{C}\right)$ is satisfied.

In practice, it is enough to derive a sufficient condition such that $(\mathscr{C})$ is satisfied. The advantage of doing this is that we can remove the non-singularity assumption of $\Theta_{k}$, which is clearly not satisfied if $Y_{k}$ is singular.

Theorem 3.2

Assume that $\Theta_{k} \Lambda_{k}-\Lambda_{k}^{-\mathrm{T}} \Phi_{k}$ is non-singular. Then the set of complex conjugate numbers $\left\{\mu_{j}\right\}_{j=1}^{k}$ satisfies condition $(\mathscr{C})$ if $\Theta_{k} \Sigma_{k}-\Lambda_{k}^{-\mathrm{T}} \Phi_{k}$ is non-singular for some $\Sigma_{k} \in \mathbb{R}^{k \times k}$ with $\sigma\left(\Sigma_{k}\right)=\left\{\mu_{j}\right\}_{j=1}^{k}$.

Proof

The arguments are similar as above. Assume that there exists $\Sigma_{k} \in \mathbb{R}^{k \times k}$ with $\sigma\left(\Sigma_{k}\right)=\left\{\mu_{j}\right\}_{j=1}^{k}$ such that $\Theta_{k} \Sigma_{k}-\Lambda_{k}^{-\mathrm{T}} \Phi_{k}$ is non-singular. Define

$$
E_{k}=\left(\Sigma_{k}-\Lambda_{k}\right)\left(\Theta_{k} \Sigma_{k}-\Lambda_{k}^{-\mathrm{T}} \Phi_{k}\right)^{-1}
$$

Then we get that

$$
\left(I_{k}-E_{k} \Theta_{k}\right) \Sigma_{k}=\left(\Lambda_{k}-E_{k} \Lambda_{k}^{-\mathrm{T}} \Phi_{k}\right)
$$

Therefore, to guarantee that $(\mathcal{C})$ holds, it remains to show that $I_{k}-E_{k} \Theta_{k}$ is invertible. It is clear that $I_{k}-E_{k} \Theta_{k}$ is non-singular if and only if $I_{k}-\Theta_{k} E_{k}$ is non-singular. Now we get from (19) that

$$
I_{k}-\Theta_{k} E_{k}=\left(\Theta_{k} \Lambda_{k}-\Lambda_{k}^{-\mathrm{T}} \Phi_{k}\right)\left(\Theta_{k} \Sigma_{k}-\Lambda_{k}^{-\mathrm{T}} \Phi_{k}\right)^{-1}
$$


Hence, $I_{k}-\Theta_{k} E_{k}$ is non-singular since $\Theta_{k} \Lambda_{k}-\Lambda_{k}^{-\mathrm{T}} \Phi_{k}$ is non-singular by the assumption. This ends the proof.

In view of Theorem 3.2, we conclude that if $\Theta_{k} \Lambda_{k}-\Lambda_{k}^{-\mathrm{T}} \Phi_{k}$ is non-singular, then the condition $(\mathscr{C})$ is satisfied for almost all complex conjugate numbers $\left\{\mu_{j}\right\}_{j=1}^{k}$. Moreover, the matrix $E_{k}$ in $(\mathscr{C})$ is explicitly given by (19) whenever $\Theta_{k} \Sigma_{k}-\Lambda_{k}^{-\mathrm{T}} \Phi_{k}$ is non-singular. Most importantly, those non-singular conditions can be easily verified in numerical computation. Note that the matrix $\Gamma_{k}=\left(I_{k}-E_{k} \Theta_{k}\right)^{-1}\left(\Lambda_{k}-E_{k} \Lambda_{k}^{-\mathrm{T}} \Phi_{k}\right)$ in Theorem 2.2 is equal to $\Sigma_{k}$. Thus, if $\Sigma_{k}$ is diagonalizable, then so is $\Gamma_{k}$. We also want to point out that the choice of assigned eigenvector $x_{j}$ satisfying $P_{\mathrm{c}}\left(\mu_{j}\right) x_{j}=0$ is clearly not unique when $k>1$. It depends on the choice of the non-singular matrix $Q_{k}$ in (16). This brings up the issue of robustness in this partial pole assignment problem, which can be solved by using the method developed in Reference [2]. We leave this issue to the serious readers.

\section{NUMERICAL SIMULATION}

In this section, we present some numerical simulation results. For testing purposes, we take a set of pseudo simulation data $M, D, K$ which is modified from the real data used by the Boeing Company. The matrices $M, D$ and $K$ are all symmetric with $M \in \mathbb{R}^{42 \times 42}$ being positive definite. Therefore, the total number of eigenvalues (counting with multiplicity) of $P(\lambda)$ is 84 . Now we let $\left\{\lambda_{j}\right\}_{j=1}^{7}=\left\{\lambda_{1}, \bar{\lambda}_{1}, \lambda_{2}, \bar{\lambda}_{2}, \lambda_{5}, \lambda_{6}, \lambda_{7}\right\}$ be a set of unwanted eigenvalues and $\left\{\mu_{j}\right\}_{j=1}^{7}=\left\{\mu_{1}, \bar{\mu}_{1}, \mu_{2}, \bar{\mu}_{2}, \mu_{5}, \mu_{6}, \mu_{7}\right\}$ be a set of prescribed eigenvalues (see Table I). Their specific values are given as follows.

We now solve the PPAPFD with these two sets of data $\left\{\lambda_{j}\right\}_{j=1}^{7}$ and $\left\{\mu_{j}\right\}_{j=1}^{7}$ as in Theorem 2.3. We first check that $\left\{\lambda_{j}\right\}_{j=1}^{7}$ and $\left\{\mu_{j}\right\}_{j=1}^{7}$ satisfy the condition ( $\mathscr{C}$ ) with $E_{k}$ explicitly computed from (19). Here we simply choose $\Lambda_{k}$ and $\Sigma_{k}$ real Jordan forms. All computations are performed in Matlab version 6.0 on a Linux machine with machine precision eps $=2.2 \times 10^{-16}$. Let $\left\{\tilde{\mu}_{j}\right\}_{j=1}^{7}$ be the computed eigenvalues of $P_{\mathrm{c}}(\lambda)=\lambda^{2} M+\lambda\left(D-B G B^{\mathrm{T}}\right)+$ $\left(K-B F B^{\mathrm{T}}\right)$ which are ordered in terms of $\left\{\mu_{j}\right\}_{j=1}^{7}$. Likewise, let $\left\{\tilde{x}_{j}=Y_{7} Q_{7} e_{j}\right\}_{j=1}^{7}$ be the associated eigenvectors as described in Theorem 2.3. The relative errors of assigned eigenvalues and the corresponding residuals are given in Tables II and III, respectively. Norms of the control matrix $B$, and the gain matrices $G$ and $F$ are given in Table IV. Next we denote $\left\{\tilde{\lambda}_{j}, \tilde{y}_{j}\right\}_{j=8}^{84}$ the

Table I. Unwanted and prescribed eigenvalues.

\begin{tabular}{ccc}
\hline$j$ & $\lambda_{j}$ & $\mu_{j}$ \\
\hline 1 & $-9.819235 e-04+2.775119 e-01 i$ & $-1.326514 e+00+2.536428 e+00 i$ \\
2 & $-9.819235 e-04-2.775119 e-01 i$ & $-1.326514 e+00-2.536428 e+00 i$ \\
3 & $+1.120362 e-01+2.394095 e+00 i$ & $-2.036517 e+00+1.453622 e+00 i$ \\
4 & $+1.120362 e-01-2.394095 e+00 i$ & $-2.036517 e+00-1.453622 e+00 i$ \\
5 & $+4.030805 e+00$ & $-3.125759 e+00$ \\
6 & $+1.959541 e+00$ & $-2.987625 e+00$ \\
7 & $-1.596075 e+00$ & $-1.523467 e+00$ \\
\hline
\end{tabular}


Table II. Relative errors of assigned eigenvalues.

\begin{tabular}{cc}
\hline$j$ & $\left|\mu_{j}-\tilde{\mu}_{j}\right| /\left|\mu_{j}\right|$ \\
\hline 1 & $4.22959668964 e-11$ \\
3 & $4.7104164755 e-12$ \\
5 & $4.02219199887 e-13$ \\
6 & $3.01747588698 e-14$ \\
7 & $5.23263838572 e-14$ \\
\hline
\end{tabular}

Table III. Residuals of $\left\|P_{\mathrm{c}}\left(\tilde{\mu}_{j}\right) \tilde{x}_{j}\right\|_{2}$.

\begin{tabular}{lc}
\hline$j$ & $\left\|P_{\mathrm{c}}\left(\tilde{\mu}_{j}\right) \tilde{x}_{j}\right\|_{2}$ \\
\hline 1 & $3.44917870585 e-08$ \\
3 & $2.26316356349 e-08$ \\
5 & $9.95346232690 e-08$ \\
6 & $5.05701556690 e-09$ \\
7 & $3.65885394330 e-09$
\end{tabular}

Table IV. Norms of control matrix $B$ and gain matrices $G, F$.

\begin{tabular}{ll}
\hline$\|B\|_{2}$ & $2.19956925250 e+01$ \\
$\|G\|_{2}$ & $1.74315183490 e+05$ \\
$\|F\|_{2}$ & $2.88768974594 e+08$ \\
\hline
\end{tabular}

computed remaining eigenpairs of $P_{\mathrm{c}}(\lambda)$. Then we obtain that

$$
\max \left\{\frac{\left|\lambda_{j}-\tilde{\lambda}_{j}\right|}{\left|\lambda_{j}\right|}, j=8, \ldots, 84\right\}=5.49195428538 e-11
$$

and

$$
\max \left\{\left\|P_{\mathrm{c}}\left(\tilde{\lambda}_{j}\right) \tilde{y}_{j}\right\|_{2}, j=8, \ldots, 84\right\}=1.287576721 e-11
$$

\section{CONCLUSION}

In this paper we propose a method to solve the partial pole assignment problem by output feedback control with feedback designs for the symmetric quadratic pencil $P(\lambda)=\lambda^{2} M+\lambda D+K$. Under a suitable condition on the unwanted eigenpairs and the prescribed values (the condition $(\mathscr{C})$ ), we can design control matrix $B$ and find gain matrices $G, F$ such that the unwanted eigenvalues are moved to prescribed values and the wanted eigenpairs remain unchanged in the closed loop-pencil $P_{\mathrm{c}}(\lambda)=\lambda^{2} M+\lambda\left(D-B G B^{\mathrm{T}}\right)+\left(K-B F B^{\mathrm{T}}\right)$. Also, the eigenvectors of $P_{\mathrm{c}}(\lambda)$ associated with the prescribed values are given explicitly without calculating the kernel of $P_{\mathrm{c}}$. The novelty of our approach is that we can make change of eigenvalues in one step and the condition $(\mathscr{C})$ can be verified quite easily. Moreover, the condition $(\mathscr{C})$ is satisfied for almost all prescribed values when the unwanted eigenpairs satisfy some generic assumptions. 
For the usual partial pole assignment problem by output feedback, the input and output matrices are in general fixed. However, it seems very difficult to relocate unwanted eigenvalues to desired values while keeping all wanted eigenstructure unchanged with fixing input and output matrices. To our knowledge, there is no result in this direction. Even though the input and output matrices in our result are determined by the unwanted eigenvectors, we believe that this is just a minor trade-off in achieving a better goal, namely, reassigning the unwanted eigenvalues and keeping the wanted eigenstructure. In addition, if the number of target unwanted eigenvalues is far less than the size of the system, so is the rank of the input (or output) matrix in our method. Furthermore, the output matrix is taken to be the transpose of the input matrix. In practice, these two advantages correspond to the fact that we do not need to put too many actuators and sensors on the structure and, most importantly, these two devices can be placed at the same positions.

\section{ACKNOWLEDGEMENTS}

We would like to thank Professor Biswa Datta for bringing this problem to our attention.

\section{REFERENCES}

1. Datta BN, Elhay S, Ram YM. Orthogonality and partial pole assignment for the symmetric definite quadratic pencil. Linear Algebra and its Applications 1997; 257:29-48.

2. Lin W-W, Wang J-N. Robust partial pole assignment for the vibrating system with aerodynamic effect. Numerical Linear Algebra with Applications 2004; 11:41-58.

3. Datta BN, Elhay S, Ram YM. An algorithm for the partial multi-input pole assignment problem of a secondorder control system. Proceedings of the 35th Conference on Decision and Control, Kobe, Japan, December 1996; 2025-2029.

4. Ram YM, Elhay S. Pole assignment in vibratory systems by multi-input control. Journal of Sound and Vibration 2000; 230:309-321.

5. Carvalho J, Datta BN, Lin W-W, Wang C-S. Eigenvalue embedding in a quadratic pencil using symmetric low rank updates, NCTS Preprints in Mathematics (2001), Website: http://math.cts.nthu.edu.tw/Mathematics/ preprints/content.html

6. Minas C, Inman DJ. Matching finite element models to modal data. Transactions of the ASME 1990; 112: 84-92.

7. Zimmerman DC, Widengren M. Correcting finite element models using a symmetric eigenstructure assignment techniques. American Institute of Aeronautics and Astronautics (AIAA) Journal 1990; 28:1670-1676.

8. Datta BN, Elhay S, Ram YM, Sarkissian DR. Partial eigenstructure assignment for the quadratic pencil. Journal of Sound and Vibration 2000; 230:101-110.

9. Nichols NK, Kautsky J. Robust eigenstructure assignment in quadratic matrix polynomials: nonsingular case. SIAM Journal on Matrix Analysis and Applications 2001; 23:77-102.

10. Tisseur F, Meerbergen K. The quadratic eigenvalue problem. SIAM Review 2001; 43:235-286. 